\title{
Visual Access Formed by Architecture and its Influence on Visitors' Spatial Exploration in a Museum
}

\author{
Rui Li* \\ Assistant Professor \\ Department of Geography and Planning \\ University at Albany, SUNY
}

rli4@albany.edu

\section{Hongchun Huang}

Associate Professor

Architecture Art Department

Sichuan Fine Arts Institute

415064357@qq.com

${ }^{*}$ corresponding author

\section{INTRODUCTION}

A visitor's spatial experience in a museum is a specific type of wayfinding behavior. In general, wayfinding can be characterized into three major types: quest, commute, and explore (Allen, 1999). Quest implies that someone tries to reach an unknown destination from a familiar point of origin. Commute indicates the movement of a person between two familiar locations and is most likely a repetitive activity. Explore indicates movement with no particular goal of reaching a pre-determined location in an unknown environment, which most closely resembles the spatial experience of someone visiting a museum for the first time. Researchers are interested in visitors' spatial experiences in a museum, as it provides valuable feedback regarding organization, placement, and attraction when planning exhibitions and drawing in visitors. For example, there are existing investigations describing the influence of a museum's layout on visitors' memory of the exhibitions (Krukar, 2014). Importantly, research from the perspective of museum curatorship addresses the sense-making of visitors' exploration of museum exhibitions (Schorch, 2013). Specific to a visitor's embodied experiences, researchers like Tzortzi (2017) investigate the understanding of space by linking the spatial and visual structure of that space. Following this suggestion, this study investigates how a museum influences the visitors' exploration of space through their visual access, as shaped by the physical structure.

Studies have suggested that the physical structure of an environment can influence a person's spatial experiences. Lynch (1960), for example suggests the difficulty of a visitor's spatial experience in an environment is associated with legibility, the easiness of understanding and finding one's way in that environment. The legibility of a space is "the property of the space that allows a situated or immersed observer to understand it in such a way as to be able to find his or her way around it" (Bafna, 2003; 26). Legibility is present in
Abstract /

A visitor's experience in space is one key research topic carried out by researchers from multiple disciplines. Regarding the architecture of a museum, research has shown that it is linked to a visitor's spatial experience in that space. These spatial experiences are relating to aspects such as a visitor's memory of location, feeling in the space, and making sense of exhibitions. Following the suggestion that architecture influences a visitor's spatial experiences, we introduce a study addressing the accessibility of one's vision, named visual access, formed by the architecture and its association with visitor's exploration pattern. Additional approaches including observation and interviews were carried out to address this question about how the architecture influences visitor's exploration in a museum. Results show that direct visual access has a greater influence than physical distance in a visitor's decision for initial exploration of a museum. In addition, these results are also used to address the pattern of exploration taken by visitors.

Keywords /

spatial exploration; museum; visual access; visibility; architecture. 
three aspects. The first aspect refers to how easily a person can differentiate sections in the environment, the second to a person's access with vision in the environment, and the third to the complexity of the environment's spatial layout. In this study, we are interested in the role that visual access has in influencing a visitor's spatial exploration of a museum. We chose to address visual access in this study, not only because of the visual structure which is associated with one's spatial experiences in museums, but also because of the characteristics of a museum operating within a large space (Tzortzi, 2017); the museum space that we refer to is comprised of multiple levels. Normally, a large museum provides an open space concept, so that layout complexity within an exhibition is not particularly extreme, as the space is needed for many visitors. Hence, layout complexity can be challenging to first-time users.

Given the uniqueness of individual collections in museum exhibitions, a visitor uses these cues to move along and differentiate each space; varying noticeable elements, such as color, size, form, or architectural style, can further help wayfinders distinguish locations and between exhibits (Evans et al., 1984). Therefore, we first aim to focus on the visual access formed by the museum's physical structure, as it may have the most influence on a visitors's spatial exploration. This includes information such as the order in which collections are visited and other spatial experiences.

The rest of this paper is organized as follows: In the Related Work, we review the concept of visual access and its potential influence on a person's spatial experiences in space. In Methods, we first introduce the museum chosen as our study site and the designed approaches for our assessment. Results present the analyses of the space in terms of its visual access and our collected data in the museum. Discussion presents the role of visual access and ways to improve visitors' spatial experience. We also evaluate the limitations and suggest potential avenues for follow-ups to this work.

\section{RELATED WORK}

Architecture plays an influential role in users' spatial experiences. It is common that if a person can easily differentiate areas of an architecture, they will be able to recognize locations while finding their way to their destination. The form of architecture contributes to the degree of differentiation, so a museum where visitors can distinguish areas contributes to the legibility of the museum's overall architecture. In addition, visual access within an environment plays a critical role in the visitor's wayfinding behaviors; visual access suggests the extent of visibility that a person can reach from a single location. The higher visibility of a place, the better visual access a person has, and previous work has linked visual access with a person's spatial experiences in a place (Gärling, Lindberg, and Mäntylä, 1983; Wiener and Franz, 2005; Hölscher et al., 2009). For example, good visual access, can enable a person to immediately find an area with an overview of surroundings, while also locating a good place to hide (Wiener and Franz, 2005). It is an effective approach, as both the best hiding and viewing places are directly associated with unobstructed visual access.

In this vein, an isovist represents the visual breadth from a single vantage point in a physical environment; it considers the convex shape of a perceptible space within $360^{\circ}$ of a standing point (Benedikt, 1979). The convex polygon representing an isovist is determined by the boundary of an environment and the areas where vision is unobscured. While the original isovist concept assumes that a person would have a complete viewing angle of $360^{\circ}$, Hölscher et al. (2009) adapted the measure that only an isovist in the person's facing direction should be considered to accurately simulate a person's viewing experiences. The improved concept is considered a partial isovist, with $120^{\circ}$ representing a human's natural vision span. An isovist only represents the visual access at one vantage point, so it does not provide an overview of an entire space. Turner et al. (2001) introduced an additional component to the isovist concept, visibility graphic analysis (VGA), which assumes all possible standing points by breaking the entire environment into a series of grids. The accumulation of isovists within each individual grid forms an overall representation of visual access in a space and is therefore a more complex representation than what is produced by using a single isovist. These accumulated results tend to show areas of best and relatively poor visual access simultaneously, and studies 
have been carried out using this method to predict visitors' attention to specific exhibitions (Krukar, 2014). This work utilizes and adapts the partial isovist concept at a museum's entrance and then conducts VGA in individual exhibition halls, associating them with observations of visitor exploration and interviews.

\section{METHODS}

This study consists of analyses of both physical environments and human wayfinding behaviors. We first introduce the selected museum and continue by describing the physical environments through quantitative methods, and the design of behavioral experiments to assess wayfinding decisions.

\section{Luo Zhongli Art Museum}

Built in 2015, Luo Zhongli Art Museum was selected as the study site because it is one of the newest buildings on the authors' campus, the largest art museum in the city, and it hosts the institute's graduation exhibition each year. The graduation exhibition has been held for 13 years, 3 of which have been hosted at this location. This museum has an area of $13,000 \mathrm{~m}^{2}$, displaying some 1,567 exhibition pieces across 12 exhibition halls, all of which are open to the public. During the graduate exhibition, student work in Chinese painting, calligraphy, oil painting, engraving, sculpture, craft, environmental design, and fashion design is displayed on three floors.

The museum has a unique structure, especially with respect to its halls. For example, Halls 2, 3, and 4, located on the first floor, are integrated with the second floor via a series of ramps that lead visitors through the flow of exhibitions. During the graduation exhibition, Hall 2 is reserved for a separate purpose and is not open for public. Aside from using elevators or the stairwell, Halls 3 and 4 have ramps that visitors can use to go upstairs. The highlighted areas in Figure 1 show the exhibition halls used for the graduation exhibition, which occupies three of the four floors of the museum. The remaining areas hold the museum's permanent collection. As a temporary exhibition showing a large number of pieces, our question is whether a visitor would have the chance to explore all work, or if the museum's architecture influences a visitor's decisions.
Consequently, identified influences can serve as suggestions and be implemented in the design and organization of next year's graduation exhibition.

The investigation considers locations on specific floors, based on the museum's physical structure and the exhibition's organization. First, during the graduate exhibition only one entrance was open, so all visitors had to enter through the main entrance (see Figure 1) and proceed through security. From this location we observed the total number of visitors within a chosen time frame, and then identified the number in specific exhibition halls.

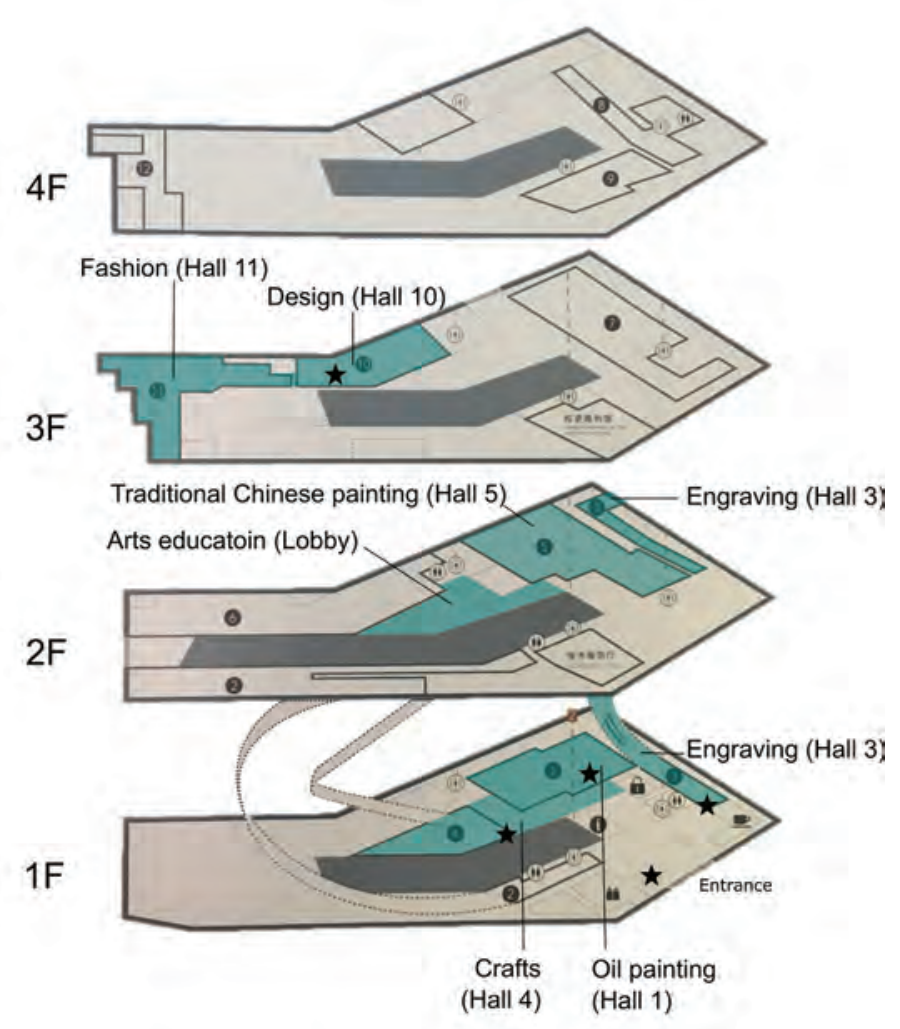

Figure 1 / Graduation exhibitions and floor plans of Luo Zhongli Art Museum (floor plan courtesy of museum). Star symbols in this figure indicate locations selected for later observations in this study.

We selected the entrances of Hall 1 (oil painting), Hall 3 (engraving), and Hall 4 (crafts) as three additional observation locations on the first floor to detect all possible visitor flows. No observations were conducted on the second floor because visitors to Halls 3 and 4 could move along a ramp to the second floor, and 
discerning movement decisions based solely on or influenced by architecture would be difficult. On the third floor, we chose the entrance to Hall 10 (design) as our observation spot because visitors to both Halls 10 and 11 (fashion design) can enter only through that location.

\section{Spatial Analysis}

The open-source software depthmapX was used for the spatial analysis. The software was developed as a space syntax tool for quantifying built environments and associating the quantified information with human behaviors (Penn 2003). This study employed the measures of partial isovist and visibility graph analysis (VGA) in the software to simulate the visual access of a museum visitor. It is important to note that this study does not evaluate other factors, such as differentiation of environment or layout complexity, which also contribute to an environment's legibility. As visual access is associated with spatial explorations, we did not consider using environmental differentiation, as it supports recognition of space and the spatial behavior investigated in this study is free exploration, which does not require spatial familiarity to orient oneself. For example, when a visitor walks into the museum, they can choose any of the three exhibition halls on the first floor to start. Furthermore, the museum is a large open space with a relatively uncomplex layout, so first-time museum visitors would likely not get lost. As such this provides a good space to explore the visual access resulting from the museum structure.

The research methods provide quantitative analyses of built environments based on their configuration and simulation of a person's visual access. Although these methods do not provide a fully comprehensive description of the environment, they address certain aspects of the environment that is essential to a person's spatial experiences. Beyond observing visitors, this study conducted interviews, which provided not only additional validation to the results of the spatial analysis, but also additional experiential input. This complete analysis of how environmental effects impact visitors can provide a more comprehensive understanding of the museum environment and how it can be altered to promote more exploration and an overall better experience for visitors.

\section{Observations and Interviews}

Data was collected in two phases. First, observations were conducted to determine the volume of visitors at selected locations in the museum, as a way to quantitatively assess the influence architecture has on visitors' exploration. Second, interviews were conducted with randomly selected visitors. The purpose was to provide a qualitative narrative to support the findings of earlier observations. The details of both components are described below.

All observations were carried out on Tuesday, June 5, 2018 between 2:30 and 3:00 p.m. The time frame was selected based on consultations with museum staff, who reported visitor flow was steadiest on weekdays between 2:00 and 4:00 p.m. Nine student assistants, who volunteered to participate in the study, conducted observations with the authors. In the morning of the observation day, students received training about the experimental protocol from the authors, with information about the groups, procedures, and how to record observations. The purpose of the training was to distinguish visitors from non-visitors. For example, someone may walk into an exhibition hall searching for a person or the bathroom instead of coming to the specific exhibition, and they should not be counted towards the total number of visitors. Each observation group consisted of at least two assistants who received the training; group members would take turns counting and verifying whether an individual qualified as a visitor. One hour before the actual observations, all assistants met with the authors at a random location in the museum to conduct a trial observation together, ensuring that all assistants were accurately counting visitors based on the protocol. The observations lasted 30 minutes in total, with five-minute observation periods. The visitor counts for both the six periods and overall session were analyzed.

Shortly after the observations concluded the authors went to the different exhibition halls, randomly selected visitors, and asked if they were willing to be interviewed. The authors introduced the purpose of the interview, which was to understand how a visitor would explore the museum and exhibitions, as well as to collect any suggestions that could enhance their experiences. The interview addressed the following five 
questions:

1. What was your first impression of the space as soon as you walked into the Museum?

2. Which was the most impressive exhibition or location for you?

3. Which exhibition hall or location was the least impressive to you?

4. What reasoning or strategies did you take to go from one exhibition to another?

5. Do you have any suggestions to enhance your experience in the museum on your next visit?

\section{RESULTS}

We first present the output from the quantitative architectural analysis, followed by descriptions of visitor observations of the museum as they relate to the spatial analysis. Data collected from visitor interviews is presented at the end of this section.

\section{Visual Access}

The isovist concept regards a person's visual access in a given location's space as restricted by its surrounding architecture, like Figure 2 represents the partial isovist at the museum entrance. Given that a partial isovist has a $120^{\circ}$ field of view, this mapping simulates the visual access a visitor has upon entering the museum, and clearly shows that a visitor can directly see the entrance of Hall 1. Entrances to other exhibition halls on this floor, however, are not visible from this location, therefore a visitor may need to investigate further and then decide whether to visit these other exhibition halls rather than freely exploring. The structure's influence on visitor decisions is discussed in the results of visitor observations.

Figure 3 shows the VGA output for the museum entrance and selected exhibition halls; the colors from dark blue to dark red represent low to high visibility, respectively. The VGA of the museum entrance shows that once visitors walk into the museum, they have a good overview of the main lobby area, but their visual attentions is likely directed toward Hall 1, which directly faces the entrance. Visitors actually have a shorter distance to the entrance of Hall 3, however the visual access to this area is less than that of the entrance to Hall 1. Around both entrances to Hall 1 the VGA shows that a person has direct visual access to the neighboring Hall 4, which allows for its discovery if this hall was not seen from the museum's entrance.

On the third-floor, visitors can only access Hall 11 through Hall 10, so it is important to investigate this dimension and the direction of visibility. VGA shows that the visibility in Hall 10 is relatively low; due to its structure, visitors 


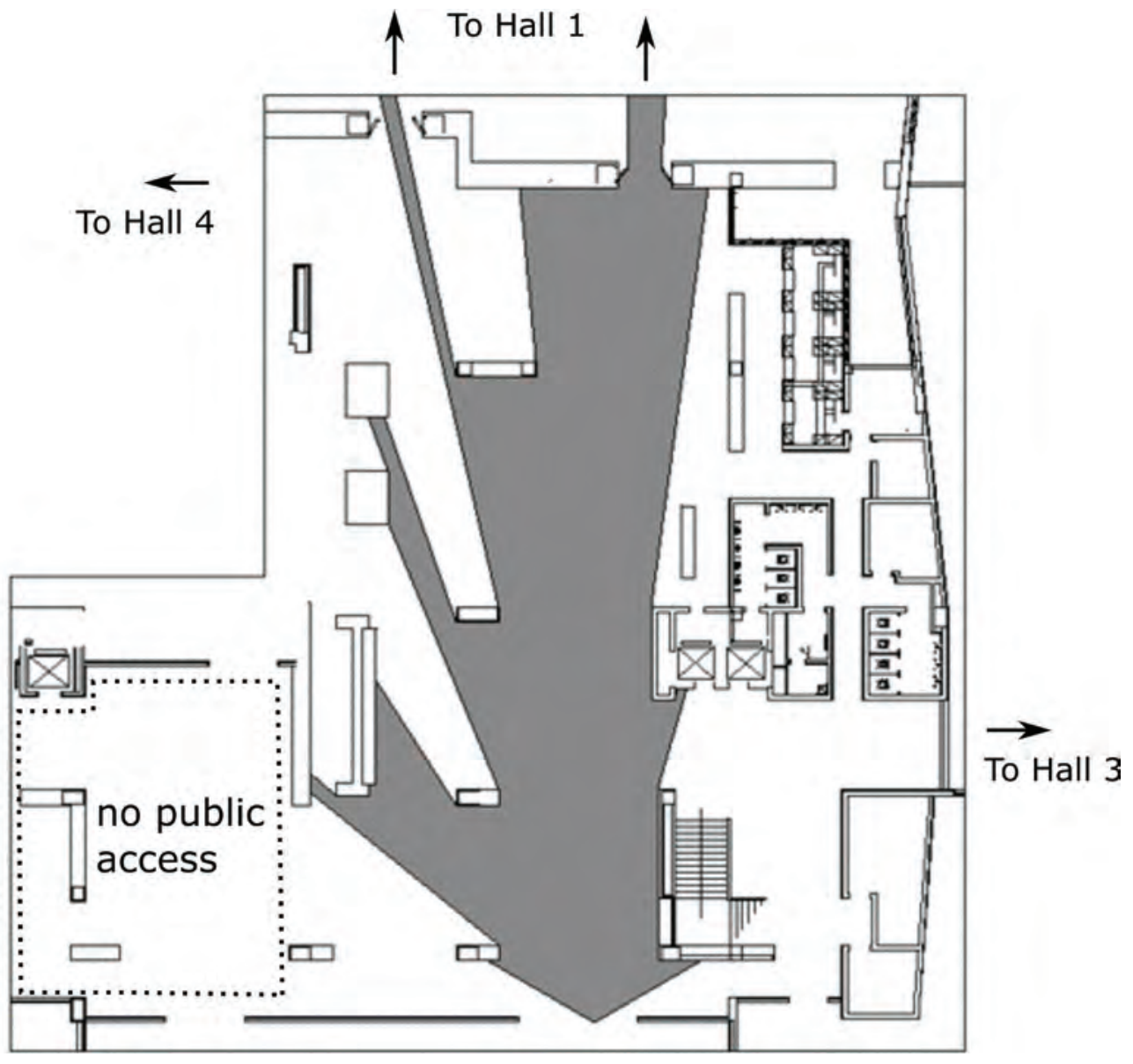

Figure 2 / Isovist simulating a visitor's visual access at the museum entrance.

are not able to see the entrance to Hall 11 until they walk to the very end of this area. It is only in this rearmost location that there is slightly higher visual access from which the entrance to Hall 11 can be seen. Overall, differences among these exhibition halls demonstrate that different architectural structures in this museum reflect various levels of visibility within the museum, which may result in different visitor exploration.

\section{Observations}

The total number of visitors was summed based on a count from every five-minute interval during the 30-minute observation window. Figure 4 shows how many visitors entered each exhibition hall. Distinguishing between visitors and non-visitors was important for ensuring an accurate count. The need for this distinction was noticeable at Hall 3, as restrooms are located near its entrance. Per the experimental design, we did not count those who were looking for the restrooms, but only those who viewed the works on display in this hall. 


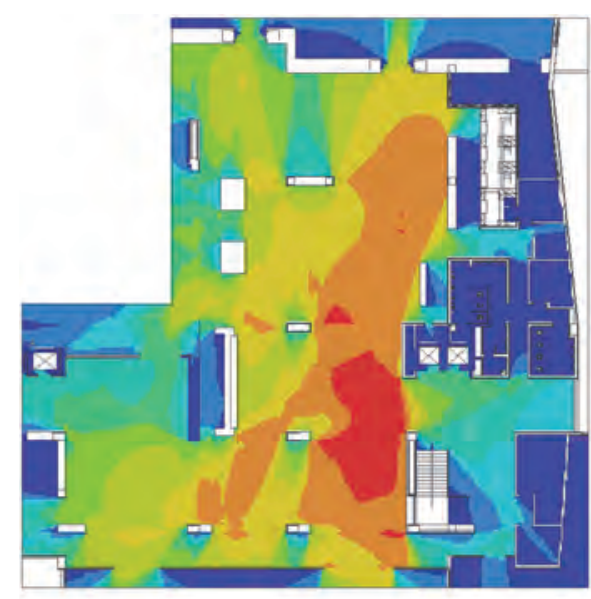

Main entrance

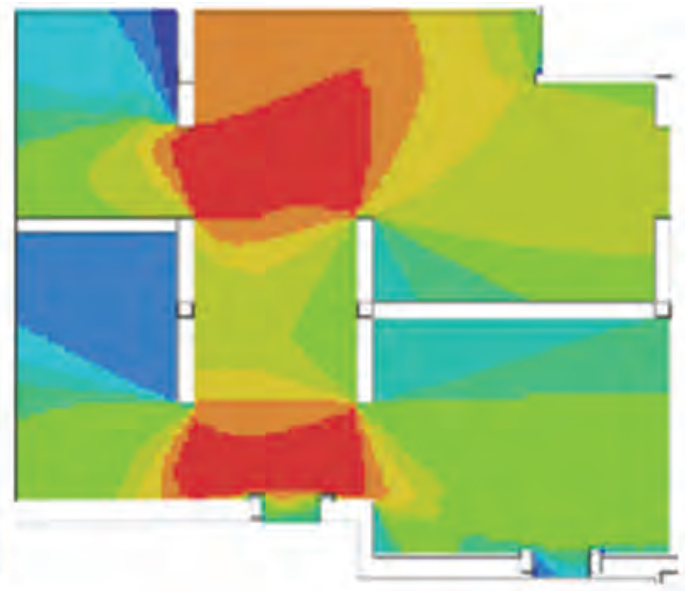

Oil painting ( $1 \mathrm{~F}$ Hall 1 )

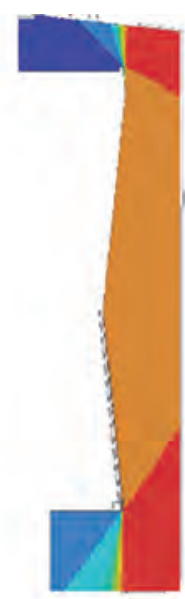

Engraving (1F Hall 3)

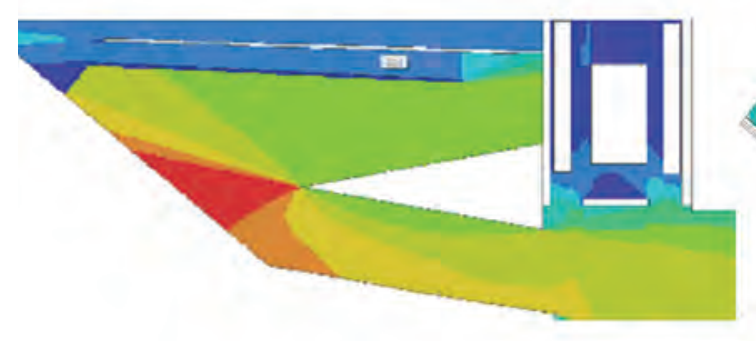

Crafts (1F Hall 4)

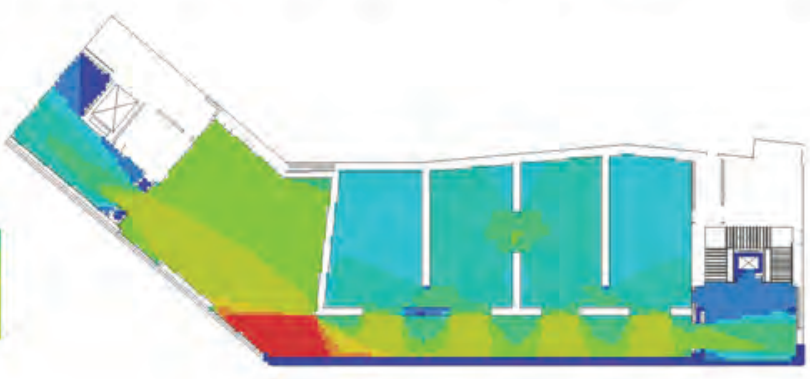

Design (3F Hall 10)

Figure 3 / VGA of observed exhibition halls showing overall visual access within each museum space.

In total, 371 visitors walked into the museum during this half-hour period, but it is important to note that some visitors may have moved very fast within the museum, visiting multiple exhibition halls, and were therefore double counted. In addition, visitors who came in before our observation window may have remained in the museum and visited some of the observed exhibition halls. Nonetheless, the volume of visitors that we counted in the exhibition halls shows the distribution of visitors within in the museum space.

Hall 4 had the highest number of visitors (249), followed by Hall 1 (184). The number of visitors to Hall 10 was the lowest (67), following behind the number of visitors to Hall 3 (123). How these numbers are associated with museum architecture is discussed after the review of the results of interviews.

\section{Interviews}

Following the observation of these exhibition halls, the authors stood at the museum's exit to seek additional information about visitors' spatial experiences randomly asking departing guests to anonymously share their experiences in the museum. Five visitors provided their thoughts in response to the authors' questions. The results here are organized by individual question. Since visitors were native 


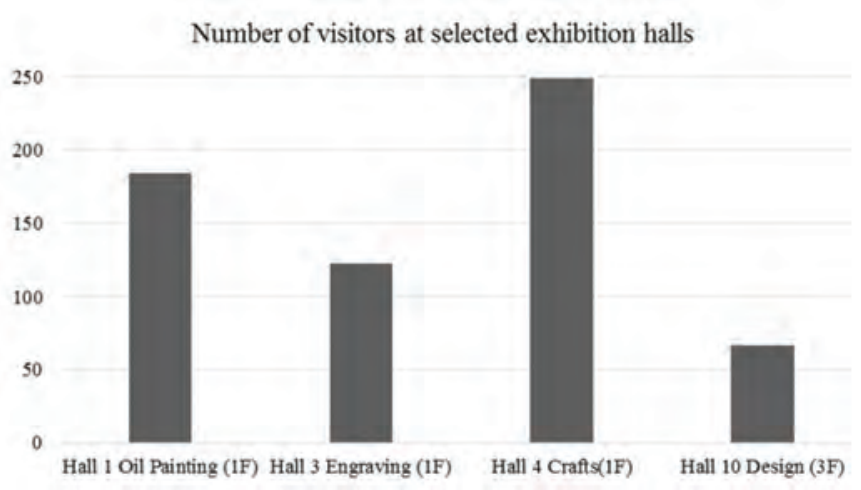

Figure 4 / Observed visitor volumes entering specific exhibition halls.

Mandarin speakers, the interviews were conducted in the visitors' native language and translated to English.

The first question asked about a visitor's initial impression of the museum as they entered the building. Many visitors mentioned that they felt the museum space was massive and they were immediately attracted to the posters highlighting the graduation exhibition. All interviewees shared that they did not know where to start, as there seemed to be many halls, but they did not find any signs or directions guiding them. In particular, three interviewees said that they picked the exhibition hall that they saw first. Interviewee 1 said, "When I just walked into the museum, I was attracted to the bold, huge poster announcing the graduation exhibition. But since I have not been in this museum before, I didn't know where to start." Interviewee 4 had a similar experience of not knowing where to start and Interviewee 2 noted that "the entire floor has been partitioned into smaller rooms with different themes. There were staircases and elevators available. But the exhibition room facing directly to the entrance was what I saw first." This opinion was closely aligned with Interviewee 3's thoughts.

The second question asked the visitors about their impression of the exhibitions. Interviewee 1 did not mention any, while Interviewee 2 stated that, "if the impression is not related to the work that I liked the most personally, I'd say that work in the hall [authors' note: Hall 1] directly facing the museum entrance and the hall right next to it in the corner [authors' note: Hall 4] as it is very spacious in this area with high ceilings and a ramp to the second floor. This was where I was most impressed." Interviewee 3 said "what impressed me the most were those works with the largest size hanging on the wall which could be viewed from multiple floors." Interviewee 4 stated that "I was mostly impressed by the oil paintings, as they are in a place that I could directly reach once I entered the museum; I just walked straight once I was in the door. Also, there were lots of visitors in this area where I spent quite some time." Based on these comments, it appears the interviewees associated their positive impressions with the architecture of the museum and the areas of direct visibility.

The third question asked which exhibit was least impactful. Two interviewees mentioned that the exhibitions of design and fashion in Halls 10 and 11, not because of the exhibitions themselves, but rather because these areas were difficult to find. For example, Interviewee 4 explicitly said that "the exhibition halls for both design and fashion were located in the wing which was the hardest to find." If I didn't remember the information from the poster that these were exhibitions of fashion and design, I would probably not have even tried to find them. It was just too hard to find." Interviewee 3 thought the general design of visitor flow was unimpressive, saying that, "There was no clear logic to view all the halls or art. I was not good at finding my way, although I didn't want to see the same work again, I found myself doing so." Interviewee 2 shared her experience in relation to the size of space, saying that "I felt least impressed by the space being separated into very small rooms and exhibitions in those small rooms didn't leave much impression on me."

The fourth question asked each interviewee regarding how they decided to explore the exhibitions and move between them. Interviewees 1 and 4 had similar strategies such as "simply follow each exhibition hall incidentally." Interviewee 2 provided more details, saying "I feel the space has some influence on my decision. If I feel a space is well connected, that will direct me to continue exploring through the space. Once I can see something in my surroundings, I'll then move on to that location." Interview 3 shared a similar strategy, that "the architecture gives me the spatial cue; I'd try to look for space that looks different so I am sure that I would not revisit a place again.” 
The last question asked the interviewees if anything could be improved to enhance their experience; only two of the five interviewees provided their thoughts, all of which addressed signage. For example, Interviewee 4 said that "I think all the exhibition halls were organized spatially with no big issues. But the signage or direction is incomplete." The other interviewee suggested that improvement to signage would enhance their ability to navigate the museum.

\section{DISCUSSION}

In this section, we aim to link the spatial analysis, visitor observation, and interviews, and to identify relationships among them, so they will not be discussed separately. Instead, all three aspects are organized around the observed exhibition halls and then summarized.

In Hall 1, the direct visual access from the museum's entrance clearly contributes to the volume of visitors. Interviewee comments further support this association. In Hall 3, the volume of visitors was the second lowest, despite that its distance to the entrance is the shortest. This is another piece of evidence supporting how direct visual access may contribute more to the visitors' urge to visit a particular hall when compared to spatial distance. The observations, as well as interviews, show that once a visitor has noticed Hall 1 through direct visual access and begins moving toward it, they then notice Hall 3 (a shorter distance), but this does not always outweigh the visitor's initial decision to move towards Hall 1. The entrance of Hall 3 is located next to the restrooms on the first floor. Although we have excluded non-visitors, some visitors may have noticed the exhibits in there after using the restrooms and then decided to enter.

Hall 4 received the greatest number of visitors, likely as the result of two factors. First, visitors potentially noticed this very open hall with high ceilings during their movement to Hall 1, so it might have been the logical place to go after viewing the oil paintings in Hall 1. Even if a visitor goes into Hall 1 without noticing Hall 4, they will have direct visual access to it no matter which door is chosen to exit Hall 1. As such, visitors were very likely to proceed to this space. The second factor is that visitors may choose to walk through Hall 4 to access the next floor, thereby viewing more work, rather than take the elevator or staircases without seeing anything. While we observed the highest number of visitors in this exhibition hall, it appeared that going here was not a visitor's first choice. Instead, its proximity to Hall 1 enabled it to receive a high volume of visitors seeking to explore the rest of the museum.

Hall 10 received the lowest number of visitors in our study; as compared to the other three halls, it had just half of the third most visited space. Interviewees who mentioned this hall were purposefully looking for it and those who made no mention of it likely had not visited it at all. The physical and organizational structures seem to play an important role in this instance, as Hall 10 is not located directly above other halls on the first or the second floor, but rather in the west wing on the third floor. Additionally, to separate the permanent collections from the graduation exhibition, the museum had blocked off space on the third floor. Therefore, this hall was only accessible through the back elevator and staircase.

If visitors took the ramp in Hall 4 to the second floor and intended to visit all exhibitions there, they would first go through the arts education exhibition and then traditional Chinese painting. Following that, they faced two possible options, one being to enter Hall 3 (engraving) on the second floor, whose ramp would return visitors to the first floor near the main entrance and the other would be to take the front elevator or staircase to the third floor. A section of the third floor hosted a permanent collection that did not provide access to the other side of that level, however, so visitors who went there would have likely returned to lower floors without visiting Hall 10. Only those who entered Hall 3 on the first floor and then moved on to the second floor would see Hall 10, as they would go through the traditional Chinese painting and then the arts education exhibition, where the back elevator or staircase would lead them to Hall 10. Even though visual access at its entrance is low, participants seemed to move along and explore the design and fashion exhibitions once they discover the exhibitions as the result of a singular direction of travel. A smaller 
portion of the visitors who entered Hall 3 might reach the exhibitions on the third floor by using an east to west exploration; the visitors counted in Hall 10 is evidence that appears to support this exploration pattern.

The visual access formed by the museum's architecture seems to play an influential role in the decision-making process around exploration. The museum selected for this study demonstrates this, as the graduation exhibition is temporary, lasting about a month before graduation, with limited signage to facilitate visitors' wayfinding. Only one poster at the entrance introduced the themes of the exhibitions, but it did not provide information of the corresponding locations. Similarly, the maps at each elevator indicated the exhibition halls by their number, but no indication of particular collections was made. Therefore, the absence of signage likely contributed to incomplete explorations. For instance, the observed number of visitors shows that direct visual access, not proximity, has a greater impact. Only a limited number of observed visitors entered a closer exhibition hall initially, then moved to the second floor, explored those exhibitions in one direction, and upon reaching the back elevator or staircase visited the third-floor exhibitions.

Visual access in the museum and its architecture for transiting visitors to the next floor generates an intuitive and exploratory flow for visitors, though only on the first two floors. Guiding visitors to the exhibition halls on the third floor remains a challenge, so identifying navigation enhancements for the third floor is worth discussing. For example, inexpensive or inobtrusive architectural changes are possible. Some additional information or temporary signs may contribute to a number of visitors on the third floor and to do that, the poster listing relevant exhibitions can be expanded to show not only the corresponding hall numbers and collections, but also indicate the floor. Furthermore, temporary maps positioned near the elevator could be easily used to show the names of collections and their corresponding locations, rather than just exhibition hall numbers as those are used only by museum management. This change would be helpful for visitors seeking a suggested exploratory route rather than free exploration. Additionally, an integrated exploration path that a visitor could make use to enhance their spatial experiences in this museum should be considered. 


\section{REFERENCES}

Allen, G.L. (1999). Spatial abilities, cognitive maps, and wayfinding: Bases for individual differences in spatial cognition and behavior. In: Wayfinding Behavior: Cognitive Mapping And Other Spatial Processes, edited by R.G. Golledge, 46-80. Baltimore: Johns Hopkins University Press.

Bafna, S. (2003). Space syntax: A brief introduction to its logic and analytical techniques. Environment and Behavior 35(1), 17-29.

Benedikt, M.L. (1979). To take hold of space: Isovists and isovist fields. Environment and Planning B: Planning and design 6(1), 47-65.

Evans, G.W., Skorpanich, M.A., Gärling, T., Bryant, K.J. and Bresolin, B. (1984). The effects of pathway configuration, landmarks and stress on environmental cognition. Journal of Environmental Psychology 4(4), 323-35.

Gärling, T., Lindberg, E. and Mäntylä, T. (1983). Orientation in buildings: Effects of familiarity, visual access, and orientation aids. Journal of Applied Psychology 68(1), 177.

Hölscher, C., Büchner, S.J., Meilinger, T. and Strube, G. (2009). Adaptivity of wayfinding strategies in a multi-building ensemble: The effects of spatial structure, task requirements, and metric information. Journal of Environmental Psychology 29(2), 208-19.

Krukar, J. (2014). Walk, Look, Remember: The influence of the gallery's spatial layout on human memory for an art exhibition. Behavioral Sciences 4(3),181-201.

Lynch, K. (1960). The Image of a City. Cambridge: MIT Press.

Penn, A. (2003). Space syntax and spatial cognition: or why the axial line? Environment and Behavior 35(1), 30-65.

Schorch, P. (2013). The experience of a museum space. Museum Management and Curatorship 28(2),193-208.

Turner, A., Doxa, M., O’Sullivan, D. and Penn, A. (2001). From isovists to visibility graphs: A methodology for the analysis of architectural space. Environment and Planning B: Planning and design 28(1), 103-21.

Tzortzi, K. (2017). Museum architectures for embodied experience. Museum Management and Curatorship 32(5), 491-508.

Wiener, J.M., and Franz, G. (2005). Isovists as a means to predict spatial experience and behavior. In: Spatial Cognition IV: Reasoning, Action, Interaction, edited by C. Freska, M. Knauff, B. Krieg-Brückner, B. Nebel, and T. Barkowsky. Berlin: Springer. 